(C) 2014 Metaphilosophy LLC and John Wiley \& Sons Ltd METAPHILOSOPHY

Vol. 45, No. 1, January 2014

0026-1068

\title{
REREADING THE KRIPKEAN INTUITION ON REFERENCE
}

\author{
BRIAN FLANAGAN
}

\begin{abstract}
Saul Kripke's thought experiments on the reference of proper names target the theory that the properties which identify a term's referent are the subject of an implicit agreement. Recently, survey versions of the experiments have been thought to show that intuitions about reference are culturally contingent. Proposing a revisionary interpretation, this article argues, first, that Kripke's Cicero/ Feynman experiment reveals that every name user knows enough to be capable of identifying the same individual as the name's most informed users. Second, the article shows that Kripke's presentation of the Gödel/Jonah experiment is ambiguous with respect to the properties attributed to the referent. Disambiguated, the experiment fails to reveal that name users may be mistaken in every unique property they attribute. Since the experiment's ambiguity is replicated in survey presentations, cross-cultural variation in survey response fails to show that intuitions about reference are culturally contingent.
\end{abstract}

Keywords: intuition, reference, descriptivism, experimental philosophy, proper names.

Appealing to our intuition, philosophers make claims about who and what terms refer to. These claims serve two purposes: to establish what a good theory of reference ought to explain, and to establish platitudes that distinguish the phenomenon of interest from (some or all) other phenomena. One condition for the achievement of either purpose is that our intuition provides an objective basis for discerning reference. The second purpose depends, additionally, on the success of a particular theory of reference, descriptivism, which states that the properties that identify a term's referent are the subject of an implicit agreement. Saul Kripke's thought experiments on the reference of proper names are variously interpreted as undermining one or both of these purposes. Traditionally, they show that a term's intuitive referent may not be identifiable through any agreement about its properties. Recently, survey versions have been thought to reveal that intuitions about reference are culturally contingent (e.g., Machery et al. 2004), leaving claims about reference without an objective basis.

Some philosophers argue that Kripke's intuitions are in fact consistent with descriptivism (e.g., Jackson 2010; Chalmers 2012). Others criticize 
the methodology of cross-cultural surveys (e.g., Ludwig 2007; Deutsch 2009; Marti 2009) and their focus on mere lay intuition (Williamson 2011; Devitt 2011). But one assumption is unquestioned: that, granted Kripke's intuitions, his experiments show only how little speakers need to know. This article challenges that assumption. I argue that, properly interpreted, the Cicero/Feynman experiment discloses that name users know enough about the referent to be capable of identifying the same individual as the name's most informed users. Second, I show that the Gödel/Jonah experiment, the subject of surveys forming the basis of the critique of intuition, features an ambiguity. Disambiguated, the experiment fails to reveal (from the armchair, at least) that name users may be mistaken in every unique property they attribute. Moreover, as the ambiguity is replicated in survey versions, a culturally diverse survey response is not clearly attributable to culturally divergent intuition. Accordingly, an interpretation of Kripke's experiments as undermining either descriptivism or the objectivity of intuition itself is premature.

\section{Cicero/Feynman: Ignorant Name Users?}

Descriptivism states that the properties that identify a term's referent are the subject of an implicit agreement. If so, the most ignorant name user ought to be capable of identifying the same individual as its most informed users. To show how ignorant name users may be, Kripke observes that, intuitively, people refer to Cicero without attributing any unique property thereto: " $[\mathrm{M}]$ ost people, when they think of Cicero, just think of a famous Roman orator, without any pretension to think either that there was only one famous Roman orator or that one must know something else about Cicero to have a referent for the name. .. . [T] he man in the street ... will say [of Richard Feynman]: well he's a physicist or something" (Kripke 1980, 80). Ask most people who Cicero was, and they will be unable to supply any unique attribute; yet they may still possess the name. As Ichikawa, Maitra, and Weatherson put it, "[I]t isn't that users think that there was only one famous Roman orator.... It's just that they don't know any more about the bearers of these names they possess" $(2012,61$; emphasis added).

Of course, it is one thing to show that speakers fail to attribute a unique property, and another to show that they fail to attribute any whatsoever. Thus, we would be hard pressed to credit possession of "Cicero" to someone who does not even know that Cicero was a classical figure. Similarly, if someone, describing "Einstein," says only, "He's a musician or something," we would be reluctant to recognise a use of the name. This reluctance suggests that we expect name users to be able to say something notable or interesting about the referent. The expectation is perceptible in Kripke's own examples. Of the causal-historical theory of reference, Kripke observes: "[A] speaker who is on the far end of this [causal] chain, 
who has heard about say, Richard Feynman, in the market place or elsewhere, may be referring to Richard Feynman even though he can't remember from whom he first heard of Feynman. He knows that Feynman is a famous physicist" $(1980,91)$. Note the care to attribute knowledge that the referent is a famous physicist. Intuitively, if all a person attributed to the referent "Feynman" were fame as a soccer player, he would not possess the name. Accordingly, Cicero/Feynman seems to reveal that we expect every name user to attribute to the referent a property distinguishing it from most other individuals. ${ }^{1}$

That a name user may identify the referent without associating a unique achievement therewith is uncontroversial. The idea of a division of linguistic labour has long been mooted as providing name users with the capacity to identify an individual through those from whom they learnt the name-for example: "[An associated description may] borrow its credentials, as a genuinely identifying reference, from another, and that from another' (Strawson 1959, 182). The difficulty is that a referent cannot be identified through those whose identity is itself forgotten or misremembered. As Kripke observes, "You may not even remember from whom you heard of Gödel" (1980, 90). ${ }^{2}$ However, a division of labour need not be composed of chains of usage. The notion of functionally specialized speakers is standardly deployed to account for natural kind terms (Putnam 1973, 704-6; Horwich 1998, 86; Harman 1999, 219-20). Hilary Putnam once ventured that proper names might equally have specialists: "[T]he fact [is] that confirmation procedures for being gold, or being aluminium, or being an elm tree, or being David are not the property of every speaker - speakers defer to experts for the fixing of reference in a huge number of cases (Putnam 1978, 114; emphasis added).

The suggestion is that a subset of name users identifies the referent on behalf of all. As Michael Devitt warns, however, expert users "cannot be identified simply as experts on the meaning of the name, on pain of circularity' $(2002,118)$. Thus, to be capable of identifying the same individuals as specialists, a speaker must be able to identify specialists in light

1 The expectation is consistent with the possibility that, in response to a question or conversation on the subject of Feynman, someone might ask: "Who is 'Feynman'?" The speaker evidently knows nothing about Feynman; certainly not that he is a famous physicist. The speaker's use of the name is significantly complicated, however, by the fact that the individual to whom the earlier speaker had intended to refer is the subject of her question. The question's answer is obvious if and only if we know to whom the earlier speaker had intended "Feynman" to refer - whether that speaker had used the name correctly is immaterial. The name thus being mentioned, rather than used, we mark its occurrence as a quotation.

${ }^{2}$ The difficulty is equally applicable to a variation on Strawson's suggestion: "[W]e can specify the property [non-experts] associate with the word 'quark'. It is having the property the group of users of the word 'quark' that they are borrowing from associate with the word "quark" (Jackson 1998a, 210). Similarly, see Jackson 1998b, 40, "Felipe Alou"; Chalmers 2002, 171, "Feynman"; and Chalmers 2012, 282, "Gödel." 
of what he or she already knows about the referent. Critically, Cicero/ Feynman suggests that the sort of properties we expect name users to attribute to the referent allow them to characterize the relevant expertise.

Recall Cicero/Feynman's indication that a name user at least attributes to the referent the property of distinction in a particular field. The attribution presupposes knowledge of what distinguishes the field and, by extension, those who work in it. Thus, the name users who attribute to Feynman only the property of achievement in science may identify scientists. Scientists, in turn, attribute unique scientific achievements to Feynman, attributions to which we expect such speakers to defer. ${ }^{3}$ Likewise, in the event that two famous scientists happened to be called "Feynman" we would expect a given name's users to attribute a subfield of achievement sufficient to distinguish its referent. Cicero/Feynman's disclosure that even the most ignorant name user can identify specialists just in light of what he or she knows about its referent implies that every name user is capable of identifying the same individual as its most informed users.

To contradict descriptivism by way of ignorant name use, Kripke must identify name users who know too little about the referent to be capable of identifying the same individual as the name's most informed users. Instead, Cicero/Feynman reveals that we expect a name user to know enough about the referent to identify expertise on the relevant individual. Of course, even a name's most informed users might be mistaken about the referent. If name users may be mistaken in every unique property they attribute, the properties that identify the term's referent are not the subject of an agreement. Kripke's second experiment is thought to confirm the antecedent.

\section{Gödel/Jonah: Mistaken Name Users?}

Taking Cicero/Feynman to show that name users may be uninformed, Kripke provides a second experiment to show that they may be misinformed. To contradict descriptivism, the experiment must reveal that even a name's most informed users could be mistaken in every unique property they attribute. Kripke describes two scenarios, one fictional: "In the case of Gödel that's practically the only thing many people have heard about him - that he discovered the incompleteness of arithmetic. Does it follow that whoever discovered the incompleteness of arithmetic is the referent of Gödel? Imagine the following blatantly fictional situation. . . . Suppose that Gödel was not in fact the author of this theorem. A man named 'Schmidt', whose body was found in Vienna under mysterious

\footnotetext{
${ }^{3}$ Even speakers who think that "Einstein's most famous achievement was the invention of the atomic bomb" (Kripke 1980, 85), attribute a field of achievement to whose practitioners we expect them to defer.
} 
circumstances many years ago, actually did the work in question. His friend Gödel somehow got hold of the manuscript and it was thereafter attributed to Gödel" (1980, 83-84). All we have heard about Gödel is that he produced the proof of incompleteness. Intuitively, however, "Gödel" does not denote the author of the theorem: "If a Gödelian fraud were exposed, Gödel would no longer be called 'the author of the incompleteness theorem' but he would still be called 'Gödel'” (Kripke 1980, 87).

Kripke's second scenario concerns the prophet Jonah. Taking the Bible's Book of Jonah to be apocryphal, Kripke notes: "If I had a suitable [history] book along with me I could quote out of it: 'Jonah, the son of Amittai, was a real prophet, however such and such and such.' There are independent reasons for thinking this was not a pure legend about an imaginary character but about a real character" (1980, 67). Though it transpires that the fish-based tale told by the Book of Jonah is untrue, it is untrue of Jonah; the Book of Jonah just led us to mistake his properties.

Attending to Kripke's presentation of the scenarios, we discover a problematic ambiguity, one replicated in survey vignettes. ${ }^{4}$ Usually, if we believe that someone achieved something, it is because we think we have reliable evidence, notably, a source that has a particular individual in mind. Ascription of a unique achievement by a particular source is itself a property, leading to our attribution to the individual of two properties, the achievement and its ascription. Kripke's presentation is silent, however, as to whether speakers believe that they have reliable evidence of the achievement's authorship and thus ascribe to the referent the unique property of being the person attributed therewith by a particular source. In the case of Kripke's Gödel, it is unclear whether we attribute authorship of the proof because we take a publication or diary to credibly ascribe it to a particular person. If so, we may correct past statements, for example, "Gödel proved incompleteness," simply because Gödel, the guy credited with proving incompleteness, did no such thing. Similarly, in the case of Kripke's Jonah, it is unclear whether we say, "Jonah did not enter a fish" simply because Jonah, the guy who was the subject of the Book of Jonah, was incapable of such feats.

Kripke later adjusts the Jonah scenario so that our only references to Jonah are those contained in the Book of Jonah itself. But this adjustment merely draws attention to the significance of the belief of name users that the author of the Book of Jonah had a particular individual in mind: "The evidence for the historicity of Jonah comes from an independent reference to him in II Kings; but such evidence could have been available in the absence of any such other references-e.g., evidence that all Hebrew legends were about actual personages" (Kripke 1980, 67). If for every other Hebrew legend we have independent evidence that it was invented

${ }^{4}$ See, e.g., those deployed in Machery et al. 2004; Lam 2010; Machery 2011; Livengood and Systema 2011; and Machery 2012; and suggested in Devitt 2011. 
about a historical personage, we may take it that the Book of Jonah was similarly about a real person. However, precisely by accepting this inference, speakers would attribute to Jonah the property of being the guy who is the subject of the Book of Jonah. If so, then, notwithstanding the invention of the fish-based tale and the lack of any other reference to him, name users are not mistaken in every unique property they attribute to Jonah. To permit the scenarios to show that name users can be so mistaken, their presentation must exclude our attribution to speakers of reliable evidence of the relevant achievement's authorship or of its attribution to a particular individual. Suitably adjusted, however, the scenarios fail to reveal that name users may be mistaken in every unique property that they attribute.

Imagine again that the only references to Jonah are those contained in the Book of Jonah itself. Should we discover evidence that Hebrew legends were never written about actual personages, we would not declare, "Jonah never visited a fish's belly" or "The Jonah of the book never existed." Instead we would say, "The Book of Jonah is a pure legend" or "There was no 'Jonah." It appears, thus, that should speakers not at least ascribe the property of being the subject of the Book of Jonah, we would not treat attributions of properties to "Jonah" as potentially mistaken. Equally, it would not seem to matter that someone was the subject of the Book of Jonah, if speakers did not actually ascribe the property. Thus, should it transpire that a folkloric figure had originally featured in a story composed about a particular peasant, we would not start to correct credulous children by saying, "Merlin was no magician." We would continue to say, "He's not a real person."

Adjusting Kripke's Gödel scenario produces a similar result. Say we lacked any credible attribution of the proof of incompleteness. Were we to learn that the proof, traditionally attributed to the mysterious Gödel, was in fact the work of the mathematician, Hans Hahn, we would not announce, "A Gödelian fraud has been exposed" or "The Gödel of the proof does not exist." Rather, we would simply treat "Gödel" as just another name for Hahn. The headlines in learned journals would read, "Gödel is Hahn!" A close actual parallel concerns the name of the mysterious author of the Iliad.

Recall the Homeric Question - that of the authorship of the oldest extant works in the Western canon. Though the classical Greeks were not credited with attributing authorship to any particular individual, "Homer" was for centuries taken to denote the poems" unique historical author. Modern Homeric scholarship has found indications that the poems were in fact the product of a long oral tradition. Were those scholars who find these indications compelling inclined to assert, "Homer did not write the epics," the name would possess a referent about whose unique properties speakers may be wholly mistaken, redeeming Kripke's strategy. As it happens, such scholars do not say such things. To the 
contrary, they construct theories of the reference of "Homer" designed to preserve the truth of the statement "Homer wrote the Iliad." 5 Thus, it has been observed that "[a]rguments in favour of one or the other option [on whether 'Homer' is a proper name or a collective noun] tend to be based... on views about the Homeric Question" (Graziosi 2002, 53). Should it somehow transpire that the ancient Athenian legislator Draco wrote the poems, scholars would presumably cease to argue that "Homer" is a noun. After all, Homer wrote the Iliad.

To contradict descriptivism, Gödel/Jonah must reveal that even a name's most informed users could be mistaken in every unique property they attribute. However, just like the treatment of "Homer" in actual scholarship, the experiment, properly interpreted, fails to do so. We observed that the vignettes used in survey versions of Gödel/Jonah replicate the ambiguity that confounds Kripke's interpretation of the experiment. It follows that the culturally diverse survey response cannot be attributed to divergent intuition ahead of divergent understandings of the scenario. Current fieldwork thus fails to show that intuition is an unreliable basis for discerning reference.

\section{Conclusion}

As the debate about Kripke's thought experiments grows in complexity, it remains commonly assumed that, granted Kripkean intuitions, they show only how little speakers need to know. This article challenges that assumption. We find that, properly interpreted, Cicero/Feynman reveals that every name user knows enough to be capable of identifying the same individual as its most informed users. Similarly, we find that Kripke's presentation of Gödel/Jonah, replicated in surveys, is ambiguous as to the existence of reliable evidence of the relevant achievement's authorship or of its attribution to a particular individual. Disambiguated, it fails to disclose the intuition that name users may be mistaken in every unique property they attribute.

Properly interpreted, then, Kripke's experiments are consistent with the purposes for which philosophers make claims about who and what terms refer to: to establish what a good theory of reference ought to explain, and to uncover platitudes that distinguish the phenomenon of interest. First, we find that survey evidence against the objectivity of intuition, the basis on which philosophers determine such claims, is flawed. Second, we find that Kripke's intuitions are in fact consistent with the theory of reference underlying the appeal to platitude, namely, that the properties that identify a term's referent are the subject of an implicit agreement.

${ }^{5}$ E.g., Graziosi 2002, 53, "a symbolic figure, the embodiment of epic poetry at large"; Nagy 1996, 90, "a metaphor that pictures the crafting of the ultimate chariot-wheel by the ultimate carpenter or "joiner"; and Durante 1957 (an assembly of poets). 
Department of Law

National University of Ireland, Maynooth

County Kildare

Ireland

brian.flanagan@nuim.ie

\section{References}

Chalmers, D. 2002. "On Sense and Intension." Philosophical Perspectives $16: 135-82$.

- 2012. Constructing the World. Oxford: Oxford University Press.

Deutsch, M. 2009. "Experimental Philosophy and the Theory of Reference." Mind and Language 24:445-66.

Devitt, M. 2002. "Meaning and Use." Philosophy and Phenomenological Research 65:106-21.

_. 2011. "Experimental Semantics." Philosophy and Phenomenological Research 82:418-35.

Durante, M. 1957. "II Nome di Omero." Rendiconti Accademia Lincei 12:94-111.

Graziosi, B. 2002. Inventing Homer: The Early Reception of the Epic. Cambridge: Cambridge University Press.

Harman, G. 1999. "Nonsolipsistic Role Semantics." In Reasoning, Meaning, and Mind, edited by G. Harman, 206-33. New York: Oxford University Press.

Horwich, P. 1998. Meaning. Oxford: Oxford University Press.

Ichikawa, J., I. Maitra, and B. Weatherson. 2012. "In Defense of a Kripkean Dogma." Philosophy and Phenomenological Research 85:5668.

Jackson, F. 1998a. "Reference and Description Revisited." Philosophical Perspectives 12:201-18.

—. 1998b. From Metaphysics to Ethics: A Defence of Conceptual Analysis. New York: Oxford University Press.

_ 2010. Language, Names, and Information. Malden, Mass.: WileyBlackwell.

Kripke, S. 1980. Naming and Necessity. Cambridge, Mass.: Harvard University Press.

Lam, B. 2010. "Are Cantonese Speakers Really Descriptivists? Revisiting Cross-cultural Semantics." Cognition 115:320-29.

Livengood, J., and J. Systema. 2011. "A New Perspective Concerning Experiments on Semantic Intuitions." Australasian Journal of Philosophy 89:315-32.

Ludwig, K. 2007. "The Epistemology of Thought Experiments: First Person Versus Third Person Approaches." Midwest Studies in Philosophy 31:128-59. 
Machery, E. 2011. "Variation in Intuitions About Reference and Ontological Disagreements." In A Companion to Relativism, edited by S. D. Hales, 118-36. New York: Blackwell. 54.

Machery, E., R. Mallon, S. Nichols, and S. Stich. 2004. "Semantics, Cross-Cultural Style.” Cognition 92:1-12.

Marti, G. 2009. "Against Semantic Multi-Culturalism." Analysis 69:4248.

Nagy, G. 1996. Homeric Questions. Austin: University of Texas Press.

Putnam, H. 1973. "Meaning and Reference." Journal of Philosophy 70:699-711.

- 1978. Meaning and the Moral Science. London: Routledge and Kegan Paul.

Strawson, P. 1959. Individuals. London: Methuen.

Williamson, T. 2011. "Philosophical Expertise and the Burden of Proof." Metaphilosophy 42:215-29. 